\title{
Is Protocolised Weaning that Includes Early Extubation Onto Non-Invasive Ventilation More Cost Effective Than Protocolised Weaning Without Non-Invasive Ventilation? Findings from the Breathe Study
}

\author{
Iftekhar Khan ${ }^{1,2} \cdot$ Mandy Maredza $^{1} \cdot$ Melina Dritsaki $^{2}$ - Dipesh Mistry ${ }^{1} \cdot$ Ranjit Lall $^{1}$ - Sarah E. Lamb ${ }^{1,3} \cdot$ Keith Couper $^{1}$. \\ Simon Gates ${ }^{1} \cdot$ Gavin D. Perkins ${ }^{1}$. Stavros Petrou ${ }^{1,4}$. On behalf of Breathe collaborators
}

Published online: 2 April 2020

(c) The Author(s) 2020

\begin{abstract}
Background Optimising techniques to wean patients from invasive mechanical ventilation (IMV) remains a key goal of intensive care practice. The use of non-invasive ventilation (NIV) as a weaning strategy (transitioning patients who are difficult to wean to early NIV) may reduce mortality, ventilator-associated pneumonia and intensive care unit (ICU) length of stay. Objectives Our objectives were to determine the cost effectiveness of protocolised weaning, including early extubation onto NIV, compared with weaning without NIV in a UK National Health Service setting.

Methods We conducted an economic evaluation alongside a multicentre randomised controlled trial. Patients were randomised to either protocol-directed weaning from mechanical ventilation or ongoing IMV with daily spontaneous breathing trials. The primary efficacy outcome was time to liberation from ventilation. Bivariate regression of costs and quality-adjusted life-years (QALYs) provided estimates of the incremental cost per QALY and incremental net monetary benefit (INMB) overall and for subgroups [presence/absence of chronic obstructive pulmonary disease (COPD) and operative status]. Longterm cost effectiveness was determined through extrapolation of survival curves using flexible parametric modelling.

Results NIV was associated with a mean INMB of $£ 620$ (\$US885) (cost-effectiveness threshold of $£ 20,000$ per QALY) with a corresponding probability of 58\% that NIV is cost effective. The probability that NIV is cost effective was higher for those with COPD (84\%). NIV was cost effective over 5 years, with an estimated incremental cost-effectiveness ratio of $£ 4618$ (\$US6594 per QALY gained).

Conclusions The probability of NIV being cost effective relative to weaning without NIV ranged between 57 and $59 \%$ overall and between 82 and $87 \%$ for the COPD subgroup.
\end{abstract}

Electronic supplementary material The online version of this article (https://doi.org/10.1007/s41669-020-00210-1) contains supplementary material, which is available to authorized users.

Iftekhar Khan

i.khan.2@warwick.ac.uk

Stavros Petrou

stavros.petrou@phc.ox.ac.uk

1 Warwick Clinical Trials Unit, University of Warwick, Coventry CV4 7AL, UK

2 Centre for Statistics in Medicine, University of Oxford, Oxford OX3 9DU, UK

3 Nuffield Department of Orthopaedics, Rheumatology and Musculoskeletal Sciences, University of Oxford, Oxford OX3 9DU, UK

4 Nuffield Department of Primary Care Health Sciences, University of Oxford, Radcliffe Observatory Quarter, Woodstock Road, Oxford OX2 6GG, UK

\section{Introduction}

Optimising techniques to wean patients from invasive mechanical ventilation (IMV) remains a key goal of intensive care practice $[1,2]$. To date, no definitive guidelines exist on the best approach to use in the intensive care unit (ICU) [2]. Evidence from structured and systematic and literature reviews of studies, including randomised controlled trials (RCTs) suggests that the use of non-invasive ventilation (NIV) as a weaning strategy (transitioning patients who are difficult to wean to early NIV) may reduce mortality, ventilator-associated pneumonia and ICU length of stay, although this beneficial effect may be limited to patients with chronic obstructive pulmonary disease (COPD) [3, 4, 8]. 


\section{Key Points for Decision Makers}

Early extubation to non-invasive ventilation (NIV) did not shorten time to liberation from ventilation.

However, the probability of NIV being cost effective relative to weaning without NIV was modest: between $57 \%$ and $59 \%$.

For patients with chronic obstructive pulmonary disorder, the probability of cost effectiveness of NIV was much higher (82-87\%).

Future trials with extended follow-up are needed to reduce uncertainty surrounding the long-term cost effectiveness of NIV.

In light of current clinical practices [2], the findings of existing trials are of limited generalizability to clinical settings across a number of industrialised countries as treatment pathways for COPD exacerbations vary across settings and over time. Whereas patients with respiratory failure would have previously received IMV, in the UK for example, this is now largely reserved for patients who fail a trial of NIV. Furthermore, few published studies have reported the impact of NIV on health-related quality of life (HRQoL) outcomes [3] or economic costs associated with NIV in critical care settings [5, 6]. Where these have been reported, sample sizes are small [6] or focus has solely been on patients with an exacerbation of COPD. Importantly, only one study, conducted in Canada, has estimated the cost effectiveness of NIV as a weaning strategy in patients with COPD [7], whereas, to our knowledge, no study has estimated its cost effectiveness in patients within the ICU presenting with or without COPD.

It is crucial to evaluate the cost effectiveness of NIV before its use can be considered more widely. We therefore present a health economic evaluation from a multicentre RCT comparing protocolised weaning that includes early extubation onto NIV versus weaning without NIV (Breathe study; ISRCTN 15,635,197).

\section{Materials and Methods}

\subsection{Trial Background}

Details of the design and clinical outcome measures for the Breathe study are reported elsewhere [8]. Briefly, patients aged $\geq 16$ years recruited from 41 UK critical care units were eligible for randomisation if they had received IMV for over $48 \mathrm{~h}$, were ready to wean and had failed a spontaneous breathing trial (SBT). Patients were randomised to receive either protocolised weaning with extubation to NIV (noninvasive weaning) or protocolised weaning via IMV with daily SBTs (invasive weaning). NIV refers to the delivery of mechanical ventilation without the need for an endotracheal airway. Positive pressure ventilation is delivered to the patient through the mouth or nose via an interface such as a mask or helmet.

Clinicians were permitted to use one of three types of SBT in accordance with local unit practices: a T-piece trial, use of continuous positive airway pressure (CPAP) or low-level pressure support $\left(5-7 \mathrm{~cm} \mathrm{H}_{2} \mathrm{O}\right)$. A T-piece trial involves the patient breathing spontaneously through their endotracheal tube, with the appropriate inspired oxygen concentration being maintained by a cross-flow device (T-piece). CPAP involved leaving a standing pressure of 5-10 $\mathrm{cm} \mathrm{H}_{2} \mathrm{O}$ delivered via the ventilator at the top of the endotracheal tube but with no assistance on inspiration. A low-level pressure support trial provided 5-7 $\mathrm{cm} \mathrm{H}_{2} \mathrm{O}$ inspiratory assistance.

Each SBT was scheduled to last for at least $30 \mathrm{~min}$ and could be increased up to $120 \mathrm{~min}$ in patients considered to be at higher risk of re-intubation (e.g. prolonged ventilation, past history of COPD, heart failure). During SBTs, patients were closely monitored for the following signs of distress or fatigue: heart rate $>20 \%$ of baseline or $>140$ beats $\mathrm{min}^{-1}$; systolic blood pressure $>20 \%$ of baseline or $>180 \mathrm{mmHg}$ or $<90 \mathrm{mmHg}$; cardiac arrhythmias; respiratory rate $>50 \%$ of baseline value or $>35 \mathrm{~min}^{-1}$, respiratory rate $(\mathrm{min}) /$ tidal volume $(\mathrm{L})>105 \mathrm{~min}^{-1} \mathrm{~L}^{-1}$; arterial blood gases; clinical assessments such as agitation, anxiety or depression. A patient was considered to pass the SBT if no signs of distress or fatigue developed. A patient who displayed any sign of distress or fatigue was judged to have failed the SBT.

Clinicians were provided with information about the Walsh criteria, which were suggested as guidance to indicate when the patient was ready to commence weaning [9]. The Walsh criteria recommend that meeting all the following conditions indicates readiness for weaning: cooperative and pain free; good cough; $\mathrm{PaO}_{2}: \mathrm{FiO}_{2}$ (ratio of arterial oxygen partial pressure to fractional inspired oxygen) $>24 \mathrm{kPa}$; positive end-expiratory pressure $<10 \mathrm{~cm} \mathrm{H}_{2} \mathrm{O}$; haemoglobin $>7 \mathrm{~g} \mathrm{dL}^{-1}$; axillary temperature $36-38.5^{\circ} \mathrm{C}$; vasoactive drugs reduced or unchanged over previous $24 \mathrm{~h}$; and spontaneous ventilatory frequency $>6$ breaths per minute. Heated humidified oxygen (on both invasive ventilation and NIV) was not mandated or recommended in the study protocol but could be used at clinician discretion in accordance with local unit policy.

A sample size of 364 (90\% power, two-sided 5\% type I error), allowing for a $23 \%$ dropout rate, was required to detect a clinically meaningful median difference of $24 \mathrm{~h}$ 
between the non-invasive and invasive group for the primary outcome of time to liberation from ventilation.

\subsection{Study Perspective and Time Horizon}

The primary economic analysis was undertaken from the perspective of the UK National Health Service (NHS) and personal social services (PSS) [10]. The time horizon for the within-trial economic evaluation was limited to a follow-up period of 6 months. In addition, a 5-year time horizon was considered for a long-term cost-effectiveness analysis. A discount rate of $3.5 \%$ per annum was applied to both costs and effects during years $2-5$ for the longer-term cost-effectiveness analysis [10].

\subsection{Measurement and Valuation of Resource Use}

Resource use data were collected from randomisation to 6 months post-randomisation using case report forms (for initial hospitalisations) and participant questionnaires. The resource and cost components associated with the intervention were aggregated into five groups:

1. intensive care support, including organ support, level of care and use of sedatives,

2. tracheostomies,

3. use of high-cost antivirals and antifungals or other highcost drugs in the ICU,

4. hospital care between ICU discharge and hospital discharge,

5. use of any emergency transport to transfer patients between hospital sites.

For critical care stays, healthcare resource groups (HRGs) were assigned according to the maximum number of organ systems supported daily during each stay. Critical care HRGs value standard resource expenditures (e.g. staffing, consumables, diagnostics), such that high-cost drugs and interventions were separately collected and valued to derive a total overall critical care cost. Following critical care discharge, the costs of step-down care were based on number of days spent in each step-downward/facility (until death or discharge) multiplied by the respective per diem cost for that level of care [11]. Further details of healthcare resource use can be found in Table 1 in the electronic supplementary material (ESM) and include the number of organs supported, the number of days in ICU, highest level of care and details of tracheostomies, antifungal/antiviral use, inpatient and outpatient care, residential care services, community health and social care, frequency of prescription medications, equipment and aids and additional health resource used by patient or carer/supporter.
Differences in resource use between the two intervention groups for the period between randomisation and hospital discharge were determined by (1) comparing the number of days that patients had two or more organs supported while in the ICU (or alternatively, days in level 3 care); (2) comparing differences in overall length of stay in the ICU; and (3) comparing proportions of patients who required tracheostomies, used high-cost antifungals, used high-cost antivirals or needed emergency transport for transfers between hospitals. Patients requiring support for two or more organs and those receiving advanced respiratory support alone were considered to receive level 3 care [12].

Broader health and PSS resource use data (e.g. hospital readmissions, contacts with community health and social care professionals, medication use) were collected at 3 and 6 months post-randomisation using postal questionnaires completed by participants or their primary carers. We also collected data on direct non-medical costs (including travel expenses) incurred by patients and their caregivers, days off work and loss of earnings. Resource use values were converted into costs by applying unit costs obtained from key UK national databases [13-20] (Table 2 in the ESM).

All costs were expressed in £ sterling (year 2015-2016 values). Where appropriate, costs were inflated to year 2015-2016 values using the NHS Hospital and Community Health Services Pay and Prices index [21].

\subsection{Measurement and Valuation of Health Outcomes}

The primary outcome for the economic evaluation was the quality-adjusted life-year (QALY) [10]. HRQoL was assessed using the three-level EuroQol 5-Dimensions (EQ5D-3L) [22] at 3 and 6 months post-randomisation. The EQ-5D-3L descriptive system consists of five dimensions (mobility, self-care, usual activities, pain/discomfort and anxiety/depression), each divided into three ordinal levels: (1) no problems, (2) some or moderate problems, and (3) severe or extreme problems. The UK time trade-off tariff was applied to each set of responses to generate an EQ5D-3L utility score for each participant [23]. Given the challenges of collecting baseline data from patients in critical care settings, we assumed in the baseline analysis that the baseline utility value was -0.402 , the value assigned by the EQ-5D-3L tariff to an unconscious health state [24]. QALY values for each patient were calculated as the area under the baseline-adjusted utility curve [25] assuming a fixed baseline of -0.402 (equivalent to an unconscious health state) and using linear interpolation between baseline and followup utility scores. QALYs were also derived from 6-Dimension Short-Form survey (SF-6D) utilities (UK tariff), generated from responses to the SF-12 as a sensitivity analysis [26], assuming a baseline utility value of zero. Patients who 
Table 1 Economic costs for complete cases for entire follow-up period, by trial allocation and cost category (£; year 2015-16 values)

\begin{tabular}{|c|c|c|c|c|c|}
\hline Evaluation period by cost type & Non-invasive $^{\mathrm{a}}$ & Invasive $^{\mathrm{a}}$ & Mean difference & $p$ value & Bootstrap 95\% CI \\
\hline Randomisation to hospital discharge & $n=182$ & $n=182$ & & & \\
\hline Intensive care support ${ }^{\mathrm{b}}$ & $20,509.7 \pm 1762.56$ & $22,018.9 \pm 1685.17$ & -1509.2 & 0.5515 & -5533.9 to 2586.3 \\
\hline Tracheostomy & $980.9 \pm 131.08$ & $1254.6 \pm 141.7$ & -273.7 & 0.1570 & -584.1 to 57.2 \\
\hline Antivirals/antifungals & $455.7 \pm 194.91$ & $758.2 \pm 219.66$ & -302.5 & 0.3036 & -768.1 to 215.8 \\
\hline Hospital care between ICU and hospital discharge & $7694.5 \pm 695.69$ & $7967.0 \pm 1038.27$ & -272.5 & 0.8275 & -2511.2 to 1682.5 \\
\hline Transfer by ambulance/NHS transport & $56.0 \pm 7.97$ & $53.1 \pm 7.82$ & 2.87 & 0.7971 & -16.1 to 20.7 \\
\hline Total NHS and PSS costs & $29,696.8 \pm 2069.8$ & $32,051.8 \pm 2204.56$ & -2355.0 & 0.4472 & -7291.5 to 2750.0 \\
\hline $\begin{array}{l}\text { Hospital discharge to } 3 \text { months post - randomisa- } \\
\text { tion }\end{array}$ & $n=130$ & $n=131$ & & & \\
\hline \multicolumn{6}{|l|}{ Health and social care resource use } \\
\hline Hospital inpatient care & $686.8 \pm 163.83$ & $381.8 \pm 123.63$ & 305.0 & 0.1387 & -30.0 to 642.2 \\
\hline Hospital outpatient care & $130.7 \pm 25.95$ & $86.7 \pm 17.41$ & -4.0 & 0.1602 & -5.2 to 97.3 \\
\hline Residential care & $158.6 \pm 55.55$ & $43.2 \pm 22.64$ & 115.4 & 0.0560 & 20.1 to 219.7 \\
\hline Community health and social care & $280.7 \pm 46.03$ & $301.3 \pm 75.31$ & -20.6 & 0.8157 & -171.3 to 114.3 \\
\hline Medications & $67.8 \pm 21.23$ & $139.5 \pm 36.84$ & -71.7 & 0.0935 & -141.4 to -4.7 \\
\hline Equipment and aids & $32.2 \pm 8.79$ & $13.9 \pm 4.71$ & 18.3 & 0.0679 & 2.8 to 35.5 \\
\hline Total NHS and PSS costs & $1356.8 \pm 196.32$ & $966.4 \pm 151.99$ & 390.4 & 0.0836 & -47.1 to 775.7 \\
\hline \multicolumn{6}{|l|}{ Broader societal costs } \\
\hline Additional $^{\mathrm{c}}$ & $108.6 \pm 33.02$ & $297.7 \pm 129.15$ & -189.1 & 0.0926 & -324.6 to -4.2 \\
\hline Equipment and aids (private and charity) & $29.3 \pm 10.53$ & $158.3 \pm 120.1$ & -129 & 0.2854 & -259.3 to 131.4 \\
\hline Total broader societal costs & $137.9 \pm 22.47$ & $456.0 \pm 93.68$ & -318.1 & 0.0012 & -434.1 to -158.4 \\
\hline Total societal costs & $1104.3 \pm 179.61$ & $1812.8 \pm 202.29$ & -708.5 & 0.0004 & -1108.2 to -308.4 \\
\hline $\begin{array}{l}3 \text { months post-randomisation to } 6 \text { months post- } \\
\text { randomisation }\end{array}$ & $n=129$ & $n=127$ & & & \\
\hline \multicolumn{6}{|l|}{ Health and social care resource use } \\
\hline Hospital inpatient care & $788.4 \pm 203.12$ & $403.0 \pm 144.18$ & 385.4 & 0.1233 & -7.0 to 1101.3 \\
\hline Hospital outpatient care & $131.5 \pm 20.23$ & $123.0 \pm 24.85$ & 28.50 & 0.7913 & -56.8 to 105.0 \\
\hline Residential care & $264.4 \pm 140.96$ & $0.0 \pm 0.00$ & 264.0 & 0.0630 & 57.6 to 515.2 \\
\hline Community health and social care & $373.2 \pm 102.74$ & $214.5 \pm 66.54$ & 158.7 & 0.1963 & -33.2 to 362.5 \\
\hline Medications & $176.2 \pm 45.39$ & $206.0 \pm 46.65$ & -29.8 & 0.6480 & -135.9 to 78.5 \\
\hline Equipment and aids & $30.9 \pm 6.30$ & $17.4 \pm 5.25$ & -13.5 & 0.1010 & -0.27 to 26.9 \\
\hline Total NHS and PSS costs & $1764.6 \pm 258.47$ & $963.9 \pm 157.71$ & 798.6 & 0.0031 & 308.9 to 1294.7 \\
\hline \multicolumn{6}{|l|}{ Broader societal costs } \\
\hline Additional $^{c}$ & $171.8 \pm 70.17$ & $245.9 \pm 110.38$ & -74.1 & 0.3864 & -207.6 to 52.9 \\
\hline Equipment and aids (private and charity) & $25.9 \pm 9.67$ & $14.7 \pm 12.5$ & 11.2 & 0.4788 & -10.8 to 33.2 \\
\hline Total broader societal costs & $197.7 \pm 45.3$ & $260.6 \pm 66.3$ & -62.9 & 0.4336 & -173.9 to 48.1 \\
\hline Total societal costs & $1962.3 \pm 265.94$ & $1224.5 \pm 178.96$ & 737.8 & 0.2262 & -432.6 to 1928.3 \\
\hline $\begin{array}{l}\text { From randomisation to } 6 \text { months post-randomi- } \\
\text { sation }\end{array}$ & $n=129$ & $n=127$ & & & \\
\hline \multicolumn{6}{|l|}{ Initial hospitalisation costs } \\
\hline Intensive care support ${ }^{\mathrm{b}}$ & $20,815.7 \pm 2,134,027$ & $21,659.9 \pm 2059.30$ & -844.2 & 0.7761 & -5699 to 4114 \\
\hline Tracheostomy & $922.6 \pm 149.01$ & $1129.5 \pm 159.01$ & -206.9 & 0.3435 & -570.1 to 151.2 \\
\hline Antivirals/antifungals & $448.2 \pm 234.98$ & $968.8 \pm 288.76$ & -520.6 & 0.1635 & -1131.2 to 89.9 \\
\hline $\begin{array}{l}\text { Hospital care between ICU and hospital dis- } \\
\text { charge }\end{array}$ & $6613.3 \pm 707.09$ & $6914.7 \pm 896.01$ & -301.4 & 0.7921 & -2229 to 1519 \\
\hline Transfer by ambulance/NHS transport & $42.6 \pm 8.33$ & $46.1 \pm 8.57$ & -3.5 & 0.7681 & -23.3 to 16.1 \\
\hline Total NHS and PSS costs & $28,842.4 \pm 2462.57$ & $30,719.0 \pm 2501.67$ & -1876.6 & 0.5934 & -7612 to 3989 \\
\hline \multicolumn{6}{|l|}{ Health and social care resource use } \\
\hline Hospital inpatient care & $1323.6 \pm 294.88$ & $698.2 \pm 221.55$ & 625.4 & 0.0913 & 28.3 to 1236.9 \\
\hline Hospital outpatient care & $240.7 \pm 39.10$ & $188.8 \pm 34.85$ & 51.9 & 0.3222 & -33.4 to 139.6 \\
\hline
\end{tabular}


Table 1 (continued)

\begin{tabular}{|c|c|c|c|c|c|}
\hline Evaluation period by cost type & Non-invasive $^{\mathrm{a}}$ & Invasive $^{\mathrm{a}}$ & Mean difference & $p$ value & Bootstrap 95\% CI \\
\hline Residential care & $405.4 \pm 150.44$ & $41.6 \pm 21.81$ & 363.8 & 0.0180 & 132.7 to 632.6 \\
\hline Community health and social care & $613.8 \pm 129.40$ & $475.5 \pm 124.74$ & 138.3 & 0.4426 & -153.7 to 424.5 \\
\hline Medications & $226.3 \pm 59.99$ & $315.8 \pm 68.56$ & -89.5 & 0.3268 & -237.9 to 60.7 \\
\hline Equipment and aids & $59.1 \pm 10.89$ & $28.6 \pm 8.45$ & 30.5 & 0.0276 & 7.3 to 53.4 \\
\hline Total NHS and PSS costs & $2868.9 \pm 392.94$ & $1748.5 \pm 276.45$ & 1120.4 & 0.0494 & 176.5 to 1542.9 \\
\hline \multicolumn{6}{|l|}{ Broader societal costs } \\
\hline Additional $^{\mathrm{b}}$ & $171.7 \pm 67.65$ & $374.6 \pm 164.09$ & -202.9 & 0.2542 & -441.1 to -23.3 \\
\hline Equipment and aids (private and charity) & $51.2 \pm 24.45$ & $157.0 \pm 98.87$ & -105.8 & 0.2498 & -179.3 to 16.0 \\
\hline Total broader societal costs & $222.9 \pm 97.44$ & $531.6 \pm 120.33$ & -309.7 & 0.0142 & -489.5 to 272.6 \\
\hline Total societal costs & $3091.8 \pm 392.94$ & $2280.1 \pm 276.45$ & 811.7 & 0.3163 & -128.3 to 1371.6 \\
\hline Total NHS/PSS including ICU & $31,711.3 \pm 2498.47$ & $32,467.5 \pm 2550.99$ & -756.2 & 0.8321 & -6642.1 to 5245.7 \\
\hline Total societal including ICU & $31,934.2 \pm 2498.58$ & $32,999.1 \pm 2547.95$ & -1064.9 & 0.7981 & -6804.2 to 5055.9 \\
\hline
\end{tabular}

$C I$ confidence interval, ICU intensive care unit, NHS national health service, PSS Personal and Social Services

${ }^{\text {a }}$ Data are presented as mean \pm standard error of the mean

${ }^{\mathrm{b}}$ Includes organ monitoring support, level of care and antibiotics

${ }^{\mathrm{c}}$ Includes travel, childcare, income lost, housework help and laundry services costs

survived the initial hospital admission were also asked to recollect their pre-admission health state using both the EQ5D-3L and the SF-12 questionnaires.

\subsection{Missing Data}

Multiple imputation under chained equations [27] was used for missing resource use or HRQoL data, based on the tested assumption that data were missing at random. Regression models were used to estimate missing costs and QALYs at each time point, by treatment allocation, conditional on fully observed baseline variables: age, sex, randomisation centre, presence/absence of COPD, non-operative/operative status and post-SBT $\mathrm{PaCO}_{2}$. In total, 20 datasets were generated using predictive mean matching. Estimates obtained were pooled to generate mean and variance estimates for costs and QALYs in each allocation group over the trial time horizon using Rubin's rule [28]. All mean incremental costs take into account heterogeneity in baseline costs, leading to estimates of incremental costs that would have adjusted for costs arising from differences between groups in terms of severity at baseline [8].

\subsection{Analyses of Resource Use, Costs and Outcome Data}

Economic values were summarised by treatment group, resource category and assessment time; differences between groups were analysed using two-sample $t$ tests. Non-parametric bootstrapping, based on 10,000 replications $(10,000$ was expected to stabilise the confidence intervals [CIs] for point estimates), was used to assess whether differences in mean total costs between allocation groups were statistically significant. EQ-5D-3L utility scores were compared using two-sample $t$ tests.

\subsection{Cost-Effectiveness Analysis}

A cost-effectiveness analysis using individual patientlevel data was conducted. Cost-effectiveness results were expressed in terms of an incremental cost-effectiveness ratio (ICER) and calculated by dividing the difference between trial arms in mean total costs by the difference in mean total QALYs. Value-for-money assessments involved comparing the ICER value with a range of cost-effectiveness thresholds. Cost-effectiveness thresholds held by UK decision makers typically range between $£ 20,000$ and 30,000 per QALY [10].

Several types of uncertainty analyses were undertaken. Stochastic uncertainty was presented in terms of CIs, decision uncertainty was undertaken using various cost-effectiveness thresholds that determined the INMB, and uncertainty from heterogeneity was implicitly assessed through modelling the pre-specified covariates (presence/absence of COPD, operative status, baseline costs, baseline utility) considered to be related to cost-effectiveness outcomes. In addition, 10,000 estimates of incremental costs and benefits were generated through non-parametric bootstrapping to determine the level of sampling uncertainty around the ICER. The bootstrap replicates were used to populate costeffectiveness scatterplots. We also calculated the incremental net monetary benefit (INMB) of using NIV versus IMV across three cost-effectiveness thresholds: $£ 15,000$ [29], $£ 20,000$ and $£ 30,000$ per QALY gained. A positive INMB 
indicates that the intervention is cost effective compared with the alternative at the given cost-effectiveness threshold. Cost-effectiveness acceptability curves (CEACs) summarised the likelihood that NIV was cost effective as the cost-effectiveness threshold varies.

\subsubsection{Sensitivity and subgroup analyses}

Sensitivity analyses included (1) adopting a wider societal perspective encompassing direct non-medical costs incurred by trial participants and their families, and economic values placed on attributable work absences being collected in the case report form by asking patients/carers whether items such as travel cost and income lost occurred and how much cost was incurred; (2) restricting analysis to complete cases; (3) using SF-6D utility scores estimated from the SF-12 for the purposes of QALY estimation; and (4) additionally using the pre-randomisation EQ-5D-3L utility value (recalled at hospital discharge) as a covariate for the purpose of QALY adjustments. Pre-specified subgroup analyses were presence/absence of COPD and operative status.

\subsection{Longer-Term Economic Modelling}

\subsubsection{Extrapolation of Survival Data}

Long-term cost effectiveness was determined over a 5-year time horizon by extrapolating survival beyond 6 months. The 5-year point was arbitrary; but we felt that extrapolating beyond 5 years would have generated too much uncertainty. For a cost-effectiveness analysis conducted over a lifetime horizon, using data from many patients who were censored would potentially lead to highly uncertain survival rates. Observed survival curves (Fig. 1 in the ESM) showed that most deaths occurred between randomisation and hospital discharge; a 5-year time horizon therefore limited the uncertainty of the long-term cost-effectiveness results compared with modelling over a lifetime horizon.

A flexible parametric model was used to predict survival rates at each time point [30]. Flexible parametric models are commonly used in the assessment of the cost effectiveness of cancer drugs for prediction of survival beyond trial followup [31]. Three parametric models were considered: exponential, Weibull and a more flexible model using cubic splines with up to four knots (points at which splines are joined). This involved fitting survival curves using the observed survival data by modelling the background (baseline hazard) risk of death over time and the risk of death due to intervention. The choice of model selection was based on Akaike's information criterion (AIC). The parameter estimates and corresponding standard errors were reported. The observed survival data (time to death) were used along with a censoring variable, and stratified covariates were included in the model.

\subsubsection{Extrapolation of Costs and Quality-Adjusted Life-Years (QALYs)}

We adopted a conservative approach to estimating (extrapolated) longer-term costs and health utilities beyond 6 months separately for each treatment group. Costs between 6 months and 5 years post-randomisation were estimated by using the observed 3- to 6-month post-randomisation total costs but also adjusting for covariates and multiplying by the predicted survival probabilities over the 5 -year post-randomisation period. The trial-based estimates of health utilities (adjusted for covariates) were used as a basis for estimating utility values beyond 6 months.

The primary assumption for longer-term cost effectiveness was that future costs and utility patterns beyond 6 months were equal, so only predicted survival rates were likely to drive future costs and QALYs. Thereafter, several sensitivity analyses were undertaken for how future cost and utility patterns could behave, including (1) the 6-month utility values were carried forward (linear constancy); (2) utilities declined in a linear fashion; (3) utilities declined exponentially; (4) survival rates were lower in the NIV arm by $10 \%$ (justified by an examination of the plots of log-survival and hazard function); (5) future costs and utilities differed between arms based on 6-month values (carried forward); and (6) future costs were assumed equal but future utilities could differ. The estimated utility values in each of these alternative scenarios (adjusted for proportion of patients alive at the corresponding time point) were used to compute the 5-year QALY estimates. A further sensitivity analysis of longer-term cost-effectiveness outcomes adopted a societal perspective.

All statistical and cost-effectiveness analyses were undertaken using SAS $^{\circledR}$ version 9.4 on a Windows platform. A published SAS macro [31] was used for flexible parametric modelling. Reporting was made in line with the CHEERS statement [32]. The trial protocol was designed by the trial investigators and was approved by South Central C Research Ethics Committee (reference 12/SC/0515).

\section{Results}

Details of the clinical study have been reported elsewhere [8]. Briefly, 364 patients were randomised: 182 to noninvasive weaning and 182 to invasive weaning. The primary clinical endpoint showed no clinical or statistical difference in median time to liberation from mechanical ventilation: median 4.3 vs. 4.5 days; adjusted hazard ratio 
1.1; 95\% CI 0.89-1.40. Early extubation to NIV did not shorten time to liberation from any ventilation. Approximately 52 and $50 \%$ of all health resource use data were complete at 3 months for the non-invasive and invasive groups, respectively; this was 51 and $46 \%$, respectively, at 6 months (Table 3 in the ESM).

A complete QALY profile was available for about $50 \%$ of patients (179/364) (Table 3 in the ESM). Data were missing because patients either died before 3 months $(n=82)$, were not available to provide a response $(n=60)$ or withdrew from follow-up $(n=37)$.

\subsection{Resource Use and Economic Costs}

Resource use for the period between randomisation and hospital discharge was generally higher for patients allocated to the invasive group (Table 1 in the ESM). The proportion of patients who used antifungals was significantly higher in the invasive group (12\% for IMV vs. $5 \%$ for NIV; $p=0.0168$ ). Broader resource use (post-initial hospital discharge) was similar between the non-invasive and invasive groups.

The mean intervention costs from randomisation until hospital discharge were $£ 29,697$ and 32,052 for NIV and IMV participants with complete data, respectively: mean cost difference $-£ 2355 ; 95 \% \mathrm{CI}-7292$ to $2750 ; p=0.4472$ (Table 1).

The mean total NHS and PSS costs throughout the first 6 months post-randomisation were, on average, lower for the non-invasive group than for the invasive group: $£ 31,711$ versus 32,468; mean difference - 756.20; 95\% CI - 6642 to $5246 ; p=0.8321$ (Table 1). Mean societal costs were $£ 31,934$ and 32,999 , respectively; mean difference -1065 ; $95 \%$ CI -6804 to $5056 ; p=0.7981$. The wide CIs for differences in mean costs reflected the uncertainty in the estimates. Significant differences between groups in terms of baseline clinical characteristics were not observed.

\subsection{Health-Related Quality-of-Life Outcomes and QALYs}

There were no significant differences in EQ-5D-3L outcomes between the trial groups prior to hospital admission or at 3 months post-randomisation (Tables 4 and 5 in the ESM).

However, mean EQ-5D-3L utility scores among complete cases were significantly lower at 6 months post-randomisation for the NIV group ( 0.53 vs. $0.66 ; p=0.0147)$. The mean QALY value was, on average, higher for the NIV group (0.0928 vs. $0.0747 ; p=0.4522)$. The mean improvement in QALYs was because mean utility at a specific time point was based on observed cases and did not consider deaths (utility score of 0 ) and differential survival rates. Since more patients died in the invasive group, more utility scores were set to zero. Moreover, our QALY estimates are derivations over time, based on modelled estimates of mean utility at each time point, taking into account heterogeneity and missing data.

\subsection{Cost-Effectiveness Analysis}

The base-case economic evaluation, using imputed attributable costs and QALYs and covariate adjustment, indicated that-over the first 6 months-NIV was associated with a lower net cost ( $-£ 302 ; 95 \% \mathrm{CI}-5490$ to 4760$)$ and a higher net effect (0.02 QALYs; 95\% CI - 0.01 to 0.05$)$ and was therefore dominant (Table 2).

The simulated ICERs showed uncertainty largely across the north-east and south-east quadrants of the cost-effectiveness plane (Fig. 1).

The NIV protocol showed net economic gains based on INMBs of $£ 541, £ 620$ and $£ 779$, on average, at costeffectiveness thresholds of $£ 15,000, £ 20,000$ and $£ 30,000$ per QALY, respectively (Table 2). The CEAC shows that the probability that NIV is cost effective is approximately 57-59\% across cost-effectiveness thresholds (Fig. 1). INMBs were similar across scenarios considered by the sensitivity analyses, indicating that the results are robust to alternative assumptions (Fig. 2; Figs. 2 and 3 in the ESM).

\subsubsection{Subgroup Analyses}

Both presence of COPD and operative status had a notable impact on cost-effectiveness results (Fig. 2; Figs. 2 and 3 in the ESM). For patients with COPD, the probability that NIV is cost effective increased to $82-87 \%$ (Table 2). In contrast, the probability of cost effectiveness was $<30 \%$ among postoperative surgical patients. A tornado diagram displaying the impact on the INMB of variations in several inputs is provided in Fig. 5 in the ESM.

\subsection{Results of Longer-Term Cost-Effectiveness Analysis}

The base-case extrapolation analysis, based on mortality predictions using the Royston-Parmar model [3 knots; i.e. a RP(4) model] yielded mean survival times (over the 5-year time horizon) of 41.9 versus 33.3 months for the non-invasive versus the invasive group, respectively. Among the three models [exponential, Weibull and RP(4)] used to predict survival rates to 5 years (Table 7 and Fig. 1 in the ESM), the RP(4) model showed the smallest AIC value (a wellestablished metric of model fit). Extrapolated survival rate estimates were in broad agreement with those in published studies [33-35], which report 1-, 2- and 3-year survival rates of $69 \%, 50 \%$ and $47 \%$, respectively. For the invasive group, these were $65 \%, 60 \%$ and $50 \%$ at years 1,2 and 3 , 


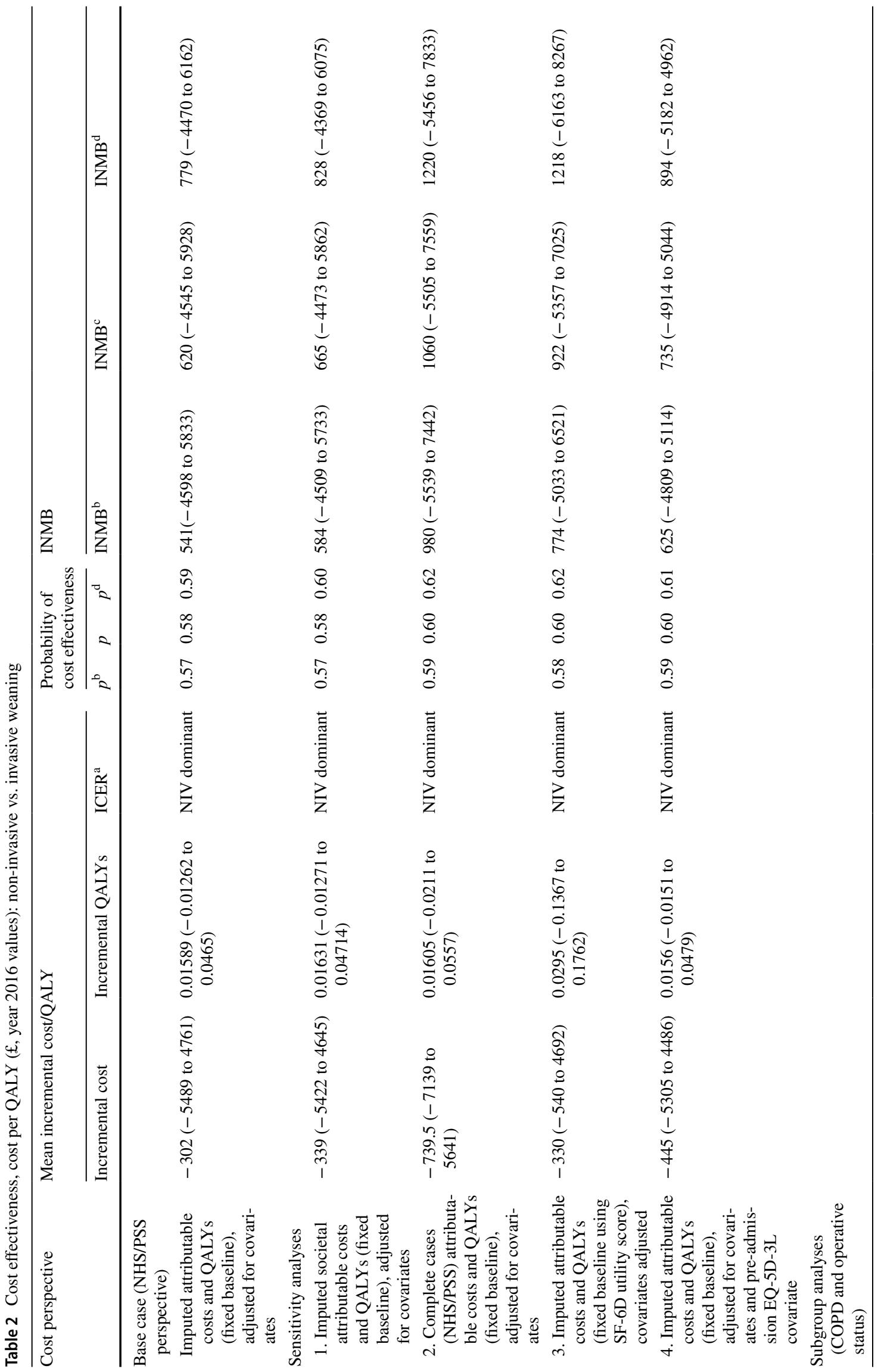




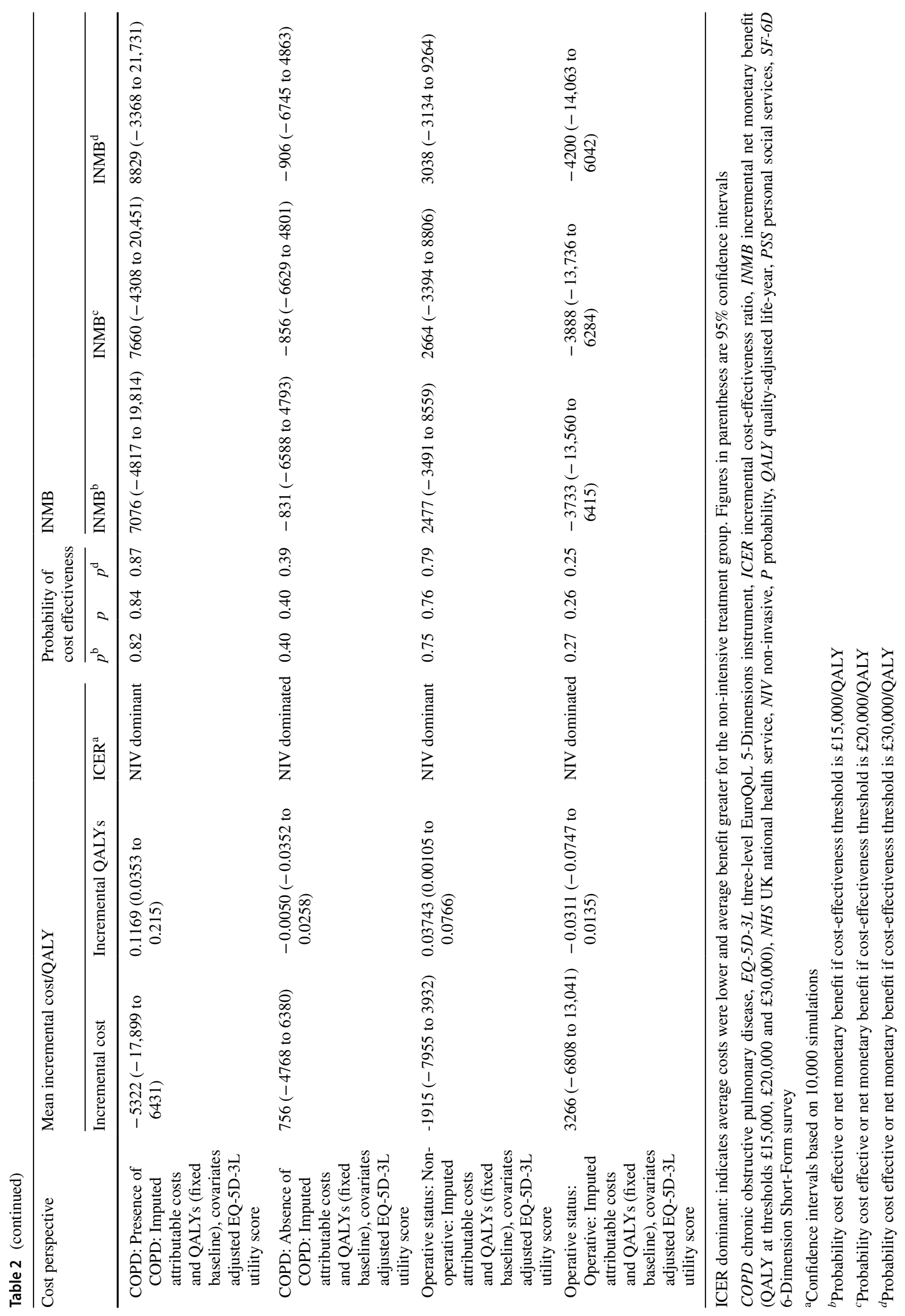


Fig. 1 a Cost-effectiveness plane, $\mathbf{b}$ cost-effectiveness acceptability curve for base case: fixed baseline utility, imputed costs, adjustment for covariates

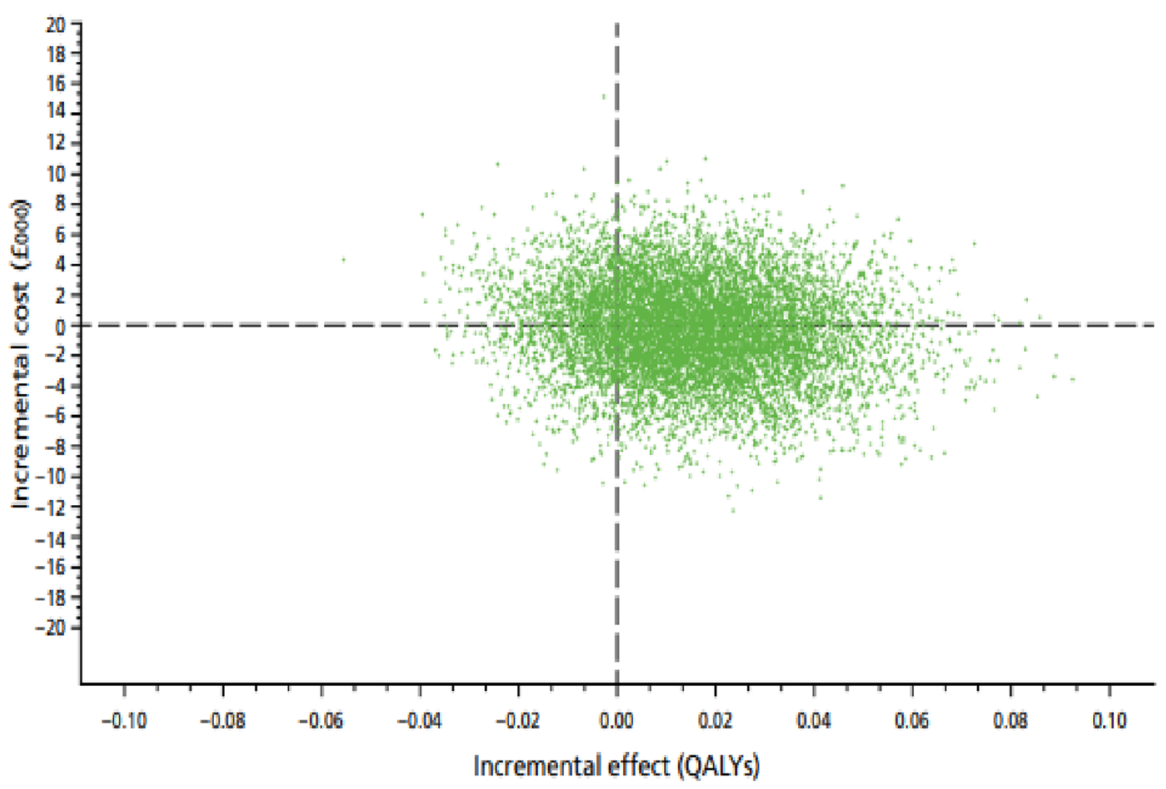

(a) Cost-effectiveness plane for base case: fixed baseline utility, imputed costs, adjustment for covariates

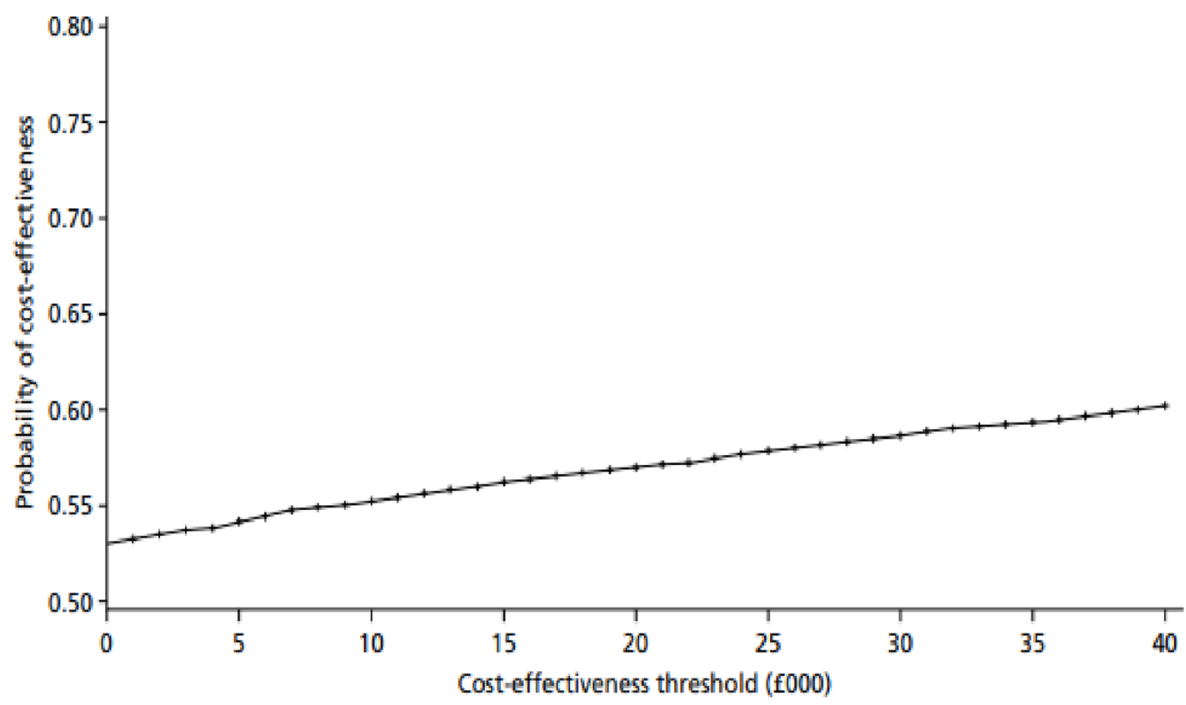

(b) Cost-effectiveness acceptability curve for base case: fixed baseline utility, imputed costs, adjustment for covariates respectively. There was no statistically significant difference between the two survival curves for survival data observed during the trial (log rank $p$ value $=0.366)$. The above models also confirmed this (Table 6 in the ESM).

The extrapolated survival to 5 years post-randomisation estimated that $67 \%$ of patients were expected to remain alive in the non-invasive group versus $45 \%$ for the invasive group. However, given the high uncertainty around these estimates as a result of extrapolation, we also conducted sensitivity analyses (Table 8 in the ESM) that included an assumption of equal future (beyond 6 months and 5 years) survival rates between arms.

Under the assumption that future costs and utility patterns beyond 6 months are equal (based on extrapolated survival data), the mean discounted expected QALYs were 2.25 (noninvasive group) and 1.82 (invasive group) (Table 7 in the ESM), resulting in an incremental QALY gain of 0.427 . The mean NHS and PSS costs over the entire 5-year period were higher in the NIV group than in the IMV group $(£ 43,759$ vs. 41,787, respectively). The 5-year ICER associated with NIV was $£ 4618$ per QALY gained (an INMB of $£ 10,838$ at 


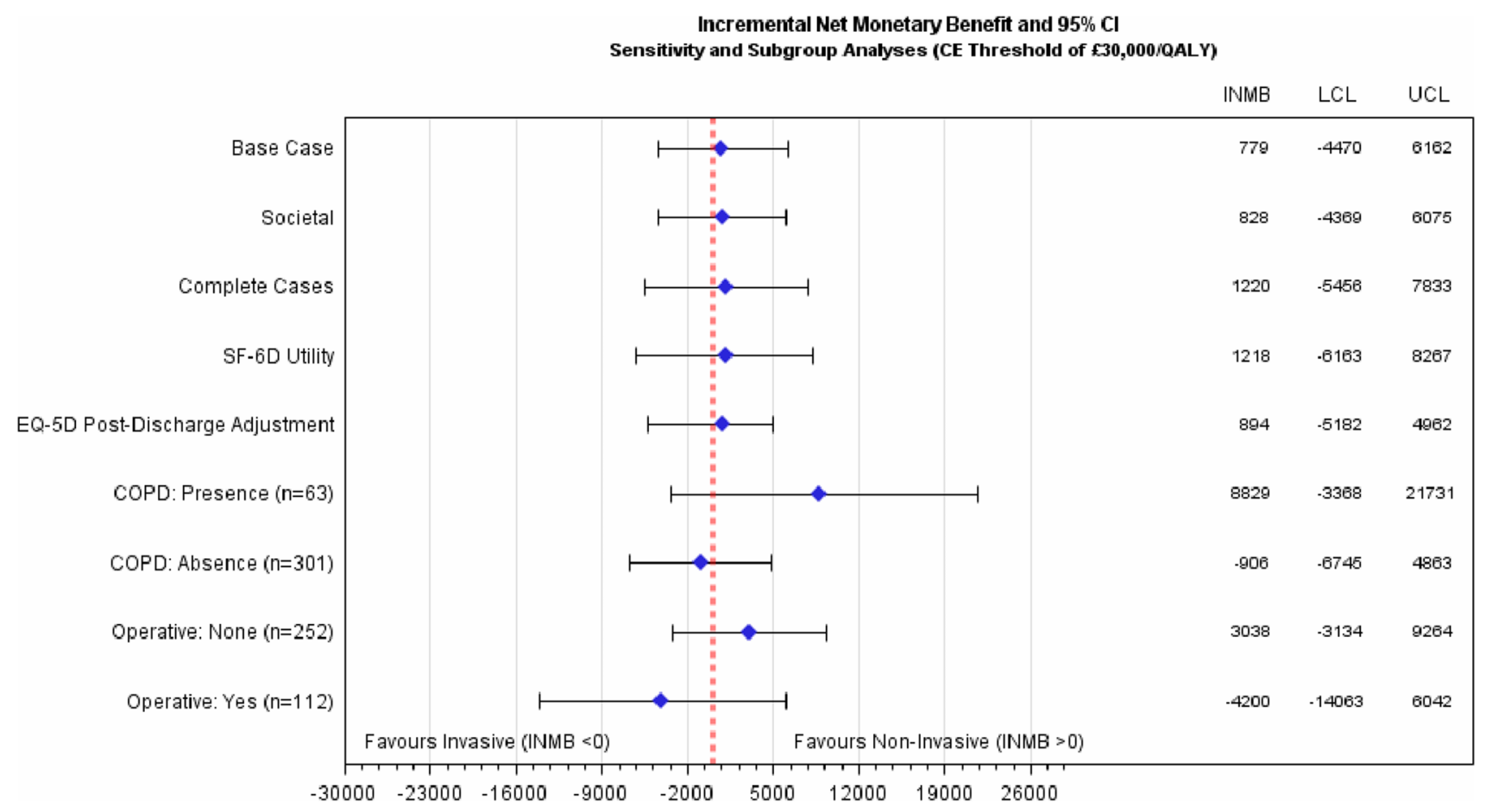

Fig. 2 Sensitivity analyses and subgroup results (cost-effectiveness threshold of $£ 30,000$ /quality-adjusted life-year)

a cost-effectiveness threshold of $£ 30,000$ ). The probability that NIV was cost effective was $>90 \%$ at a cost-effectiveness threshold of $£ 20,000$ per QALY (Fig. 7 in the ESM).

However, the sensitivity analyses indicated that the probability of cost effectiveness for NIV strongly depended on assumptions surrounding future costs, utilities and survival rates beyond 6 months (Table 8 and Figs. 4 and 6 [tornado plot] in the ESM). In particular, under the assumption of equal survival rates between 6 months and 5 years, and equal future utilities, the mean incremental costs and QALYs were (non-invasive vs. invasive group) $£ 6453$ and -0.092 (Table 8 in the ESM), respectively, yielding negative INMBs ranging between $£ 7833$ and $£ 9213$ favouring invasive weaning. This showed that the incremental QALY gains in favour of NIV were largely driven by the higher extrapolated survival rates for the NIV arm. Hence, despite predicted survival rate estimates being in broad agreement with those in published studies [33-35], the uncertainty around the long-term cost-effectiveness results should be carefully considered.

When the projected survival rates were assumed to be lower by $10 \%$ in the non-invasive weaning arm (vs. the invasive arm), the INMB fell by $>90 \%$ from $£ 10,838$ to 603 , and the expected probability of long-term cost effectiveness fell to around $79 \%$ (Table 8 and Fig. 8 in the ESM). If future survival estimates reflect patterns shown here (Fig. 1 in the ESM), and future patient costs accumulate based on patterns observed during the trial, without improvements in QALYs (Table 8 in the ESM, scenario 4), NIV is no longer be cost effective (change from INMB of $£ 10,838$ in favour of NIV to INMB of $£ 6061$ favouring invasive weaning).

\section{Discussion}

This trial-based economic evaluation showed that NIV has potential to be cost effective compared with invasive weaning; the probability of cost effectiveness ranged from 57 to $59 \%$, depending on the cost-effectiveness threshold. This is likely to be largely driven by the difference in the mean \pm standard error number of days in the ICU $(14 \pm 1.08$ vs. $15 \pm 1.12$ ) and ICU-related costs (Table 1; Table 1 and 9 in the ESM).

This finding remained robust to most sensitivity and subgroup analyses considered. The main exception related to subgroups of patients without COPD and those who required surgery, in whom IMV was the dominant strategy.

The primary clinical endpoint of time to liberation from mechanical ventilation did not show a statistical difference: median 4.3 vs. 4.5 days; adjusted hazard ratio $1.1 ; 95 \% \mathrm{CI}$ 0.89-1.40. However, a lack of difference in the time to liberation from ventilation does not imply that costs of ICU care beyond this point are no longer relevant for the purposes of cost effectiveness. In this cost-effectiveness analysis, health 
resource use and HRQoL beyond liberation from ventilation were considered, which could logically result in differing conclusions. We observed that, on average, patients in the invasive group stayed about 1 day long in the ICU than those in the non-invasive group (Table 1 in the ESM). Moreover, other costs, including broader NHS and broader societal costs, also influenced the observed cost differential in favour of NIV. In addition, improved QALYs associated with NIV were observed once deaths are accounted for (driven by more deaths in the IMV arm). The cost-effectiveness analysis considered a broader assessment of consequences than the clinical study, resulting in a conclusion that NIV weaning may be cost effective, particularly for patients with COPD.

The differences in costs and QALYs between the trial comparators were not statistically significant. However, the trial was not powered to detect such differences over a short-term time horizon. A power calculation for a future trial covering a 5-year follow-up period, using the observed results from this trial, taking into account long-term survival predictions (i.e. using mean costs and projected QALYs and their standard deviations along with a $£ 30,000$ cost-effectiveness threshold), would require a sample size of at least 215 per group (430 in total) to show with at least $80 \%$ power (5\% significance) that the INMB would be $>0$. This would be the case even if NIV weaning was more expensive by as much as $£ 2737$ (one possible scenario of the sensitivity analyses in Table 8 in the ESM). Hence, this trial provides useful data to prospectively design a future (larger) confirmatory trial with longer follow-up that could demonstrate long-term cost effectiveness. In the more optimistic scenario, where NIV was projected over 5 years to be cheaper by $£ 302$ (Table 2; Table 8 in the ESM), the sample size required would be around 120 per group (240 in total). The observed probabilities of cost effectiveness observed in this trial, although $<80 \%$, may partly be because the sample size ( $n=364$ vs. $n=430$ ) was smaller than required to demonstrate cost effectiveness [36].

Previous clinical trials have reported significant clinical benefits with the NIV protocol among patients with COPD, in contrast to studies that enrolled mixed populations [3]. However, none of those studies reported impacts on HRQoL among survivors. Therefore, our findings add new insights into available evidence, emphasising that other factors may need to be considered when deciding on optimal weaning approaches for patients in critical care settings who present without COPD.

When the benefits of NIV weaning on mortality were extrapolated to 5 years, the NIV protocol remained cost effective (5-year ICER $£ 4618 / \mathrm{Q} A L Y$ (INMB $£ 6568$ at a cost-effectiveness threshold of $£ 20,000$ ) with probability of cost effectiveness of NIV $>90 \%$ ). NIV as a bridge to liberation from mechanical ventilation could be recommended on economic grounds provided that the assumptions of constant costs and health utilities beyond the trial period continue to hold in practice. The longer-term cost effectiveness of NIV is highly dependent on assumptions surrounding costs and benefits beyond 6 months. However, we have included sensitivity analyses for various parameters (including survival rates) while noting that estimated survival rates were broadly similar to those reported in the literature [33-35]. We also suggest that additional information on longer-term outcomes is needed in future studies to reduce the uncertainty around our estimates.

To our knowledge, this is the first trial-based economic evaluation that compares the cost effectiveness of two protocolised weaning strategies for patients in the ICU. Previous studies have compared the cost effectiveness of NIV weaning with other strategies and in different countries and clinical settings. Chandra et al. [7] conducted a cost-effectiveness analysis of multiple interventions for COPD, including a comparative assessment of weaning protocols (NIV vs. weaning with invasive ventilation), within the Canadian setting, using a Markov probabilistic model. The results indicated that weaning with NIV dominated weaning with invasive ventilation; the probability of cost effectiveness for NIV weaning exceeded $99 \%$ at cost-effectiveness thresholds as low as $\$ 25,000$ per QALY gained. Nonetheless, the analysis was restricted to patients with COPD. We also considered using the data from Chandra et al. [7] as an external input but felt that long-term extrapolations of survival could be misleading or unreliable because the populations differed. Other studies reported costs associated with NIV weaning in critical care settings [5, 6] but did not report HRQoL outcomes and were either based on small sample sizes [6] or focussed solely on patients with a COPD exacerbation [37].

A strength of the Breathe trial was that it was prospectively designed for an economic evaluation using individuallevel data. Costs and outcomes were carefully considered in the trial design with a view to reaching a robust costeffectiveness conclusion based on a large sample. However, potential limitations to this analysis do exist. First, we assumed that the baseline utility value for each patient was -0.402 , the value assigned by the UK EQ-5D-3L tariff to an unconscious health state. This assumption is in keeping with broader methodological practice for trial-based economic evaluations conducted in critical care settings [38]. Moreover, we recently demonstrated that applying alternative fixed baseline utility scores generally had no effect on incremental QALY calculations [38]. Second, approximately $35 \%$ of QALY data and 6-40\% of costs (at the component level) were missing by 6 months. Had our base-case costeffectiveness analysis only considered individuals with complete QALY and cost data, we would have removed approximately $50 \%$ of patients from the analysis, which would have likely biased the results. After demonstrating that the data 
were missing at random, we used multiple imputation to 'replace' missing values to allow a comprehensive analysis using the whole dataset. Third, the modelling undertaken was constrained by assumptions concerning post6-month costs and health utilities. However, a systematic search of external studies that compared these two weaning approaches revealed that none reported long-term economic outcomes. As a result, the evidence base for extrapolating cost effectiveness is currently weak. Longer follow-up would have reduced uncertainty surrounding our long-term costeffectiveness estimates.

\section{Conclusions}

The results from the Breathe study indicated that early extubation to NIV did not shorten time to liberation from any ventilation. The probability of NIV being cost effective relative to weaning without NIV ranged between 57 and $59 \%$ and was higher for patients with COPD (82-87\%). Future trials with extended follow-up are needed to reduce uncertainty surrounding the long-term cost effectiveness of NIV.

Author Contributions Dr. IK (Senior Research Fellow, Health Economics and Associate Professor of Statistics) was a member of the TMG, was responsible for the economic analysis of the trial and wrote and reviewed the report. Prof. SP (Professor of Health Economics, co-applicant) contributed to protocol development, was a member of the TMG, was responsible for the economic analysis of the trial and wrote and reviewed the report. Dr. MM and Dr. MD (Senior Research Fellows, Health Economists) were members of the TMG and reviewed the report. Dr. DM (Senior Research Fellow, trial statistician) and Prof. RL (Principal Research Fellow, co-applicant) developed the protocol, were members of the TMG, were responsible for the statistical analysis of the trial and wrote and reviewed the report. Prof. SL (Professor of Rehabilitation, co-applicant) and Prof Simon Gates (Professor of Medical Statistics, co-applicant) developed the protocol, were members of the TMG and wrote and reviewed the report. Dr. KC (Assistant Professor) developed the protocol, was involved in data acquisition and wrote and reviewed the report. Professor GP (Chief Investigator Applicant) was involved in study design conception, developed the protocol, was a member of the TMG and wrote and reviewed the report.

Data Availability The datasets generated during and/or analysed during the current study are available from the corresponding author on reasonable request.

\section{Compliance with Ethical Standards}

Funding The Breathe study was funded by the National Institute for Health Research (NIHR) Health Technology Assessment programme (project number HTA no 10/134). The views expressed are those of the authors and not necessarily those of the NHS, the NIHR or the Department of Health and Social Care.

Conflict of Interest $\mathrm{KC}$ is supported by an NIHR post-doctoral fellowship. Gavin D. Perkins received grants and non-financial support from the Intensive Care Foundation during the conduct of the study. IK, MM, MD, DM, RL, SEL, SG and SP have no conflicts of interest that are directly relevant to the content of this article.

Ethics Approval This study was approved by the UK institutional and national research ethics committee and has been performed in accordance with the ethical standards of the Declaration of Helsinki. Informed consent was obtained from all individual participants included in the study.

Open Access This article is licensed under a Creative Commons Attribution-NonCommercial 4.0 International License, which permits any non-commercial use, sharing, adaptation, distribution and reproduction in any medium or format, as long as you give appropriate credit to the original author(s) and the source, provide a link to the Creative Commons licence, and indicate if changes were made. The images or other third party material in this article are included in the article's Creative Commons licence, unless indicated otherwise in a credit line to the material. If material is not included in the article's Creative Commons licence and your intended use is not permitted by statutory regulation or exceeds the permitted use, you will need to obtain permission directly from the copyright holder.To view a copy of this licence, visit http://creativecommons.org/licenses/by-nc/4.0/.

\section{References}

1. Bentley A: Weaning centres an unfashioanable neccessity. 2018; https://www.ics.ac.uk/ICS/Blogs/Weaning_Centres_an_unfas hionable_necessity_.aspx. Accessed May 12, 2018

2. Burns KE, Raptis $\mathbf{S}$, Nisenbaum R, et al. International Practice Variation in Weaning Critically Ill Adults from Invasive Mechanical Ventilation. Ann Am Thorac Soc. 2018;15(4):494-502.

3. Burns KEA, Meade MO, Premji A, et al. Noninvasive positive-pressure ventilation as a weaning strategy for intubated adults with respiratory failure. Cochrane Database Syst Rev. 2013;12:1465-858.

4. Glossop AJ, Shepherd N, Bryden DC, et al. Non-invasive ventilation for weaning, avoiding reintubation after extubation and in the postoperative period: a meta-analysis. $\mathrm{Br} \mathrm{J}$ Anaesth. 2012;109(3):305-14.

5. Criner GJ, Kreimer DT, Tomaselli M, et al. Financial implications of noninvasive positive pressure ventilation (NPPV). Chest. 1995;108(2):475-81.

6. Nava S, Evangelisti I, Rampulla C, et al. Human and financial costs of noninvasive mechanical ventilation in patients affected by COPD and acute respiratory failure. Chest. 1997;111(6):1631-8.

7. Chandra K, Blackhouse G, McCurdy BR, et al. Cost-effectiveness of interventions for chronic obstructive pulmonary disease (COPD) using an ontario policy model. Ont Health Technol Assess Ser. 2012;12(12):1-61.

8. Perkins GD, Mistry D, Gates S, et al. Effect of protocolized weaning with early extubation to noninvasive ventilation vs invasive weaning on time to liberation from mechanical ventilation among patients with respiratory failure: the breathe randomized clinical trial. JAMA. 2018;320(18):1881-8.

9. Walsh TS, Dodds S, McArdle F. Evaluation of simple criteria to predict successful weaning from mechanical ventilation in intensive care patients. Br J Anaesth. 2004;92(6):793-9.

10. National Institute for Health and Care Excellence (NICE). Guide to the Methods of Technology Appraisal. 2017; https://www.nice. org.uk/process/pmg9/chapter/foreword. Accessed 3 Aug 2017. 
11. Department of Health. Reference costs guidance 2015-2016. 2017; https://assets.publishing.service.gov.uk/government/uploa ds/system/uploads/attachment_data/file/497127/Reference_costs _guidance_2015-16.pdf. Accessed 3 Aug 2017.

12. Shirley P. What is the difference between ICU and HDU? BMJ. 2005;330(7499):s184.

13. Curtis LA, Burns A. Unit costs of health and social care. Canterbury: University of Kent, Personal Social Services Research Unit; 2010.

14. Curtis LA, Burns A. Unit costs of health and social care. Canterbury: University of Kent, Personal Social Services Research Unit; 2014.

15. Curtis LA, Burns A. Unit costs of health and social care. Canterbury: University of Kent, Personal Social Services Research Unit; 2015.

16. Joint Formulary Committee. British National Formulary. 2017; https://www.evidence.nhs.uk/formulary/bnf/current. Accessed 20 May 2017.

17. NHS. NHS Supply Chain 2016. 2017; https://my.supplychain.nhs. uk/catalogue. Accessed 20 May 2017.

18. NHS Improvement. NHS Reference Costs 2014 to 2015. 2017; https://www.gov.uk/government/publications/nhs-reference-costs -2014-to-2015. Accessed 18 Oct 2017.

19. NHS Improvement. NHS Reference Costs 2015 to 2016. 2017; https://www.gov.uk/government/publications/nhs-reference-costs -2015-to-2016. Accessed 18 Oct 2017.

20. Office for National Statistics. Annual Survey of Hours and Earnings. 2017; https://www.ons.gov.uk/employmentandlabourmarket /peopleinwork/earningsandworkinghours/bulletins/annualsurv eyofhoursandearnings/previousReleases. Accessed 18 Feb 2017.

21. Department of Health. Hospital and Community Health Services (HCHS) Pay and Price Inflation. 2017; https://www.info. doh.gov.uk/doh/finman.nsf/af3d43e36a4c8f8500256722005b77 f8/360a47827991d10a80258036002d8d9f/\$FILE/2015.16\%20Pay \%20\&\%20Price\%20series.xlsx. Accessed 20 May 2017.

22. van Reenen M, Oppe M. EQ-5D-3L User Guide EUROQOL. 2017; https://euroqol.org/wp-content/uploads/2016/09/EQ5D-3L_UserGuide_2015.pdf. Accessed 20 May 2017.

23. Dolan P. Modeling valuations for EuroQol health states. Med Care. 1997;35(11):1095-108.

24. Dolan P, Gudex C, Kind P, et al. The measurement and valuation of health: final report on the modelling of valuation tariffs. 2017; https://www.york.ac.uk/media/che/documents/reports/MVH\%20 First\%20Report.pdf. Accessed 20 May 2017.

25. Kind P. UK Population Norms for the EQ-5D. 2017; https://www. york.ac.uk/che/pdf/DP172.pdf. Accessed 12 May 2017.
26. Brazier J, Roberts J, Deverill M. The estimation of a preference-based measure of health from the SF-36. J Health Econ. 2002;21(2):271-92.

27. White IR, Royston P, Wood AM. Multiple imputation using chained equations: Issues and guidance for practice. Stat Med. 2011;30(4):377-99.

28. Rubin DB. Multiple imputation for nonresponse in surveys. New York: Wiley; 2004.

29. Claxton K, Martin S, Soares M, et al. Methods for the estimation of the NICE cost effectiveness threshold. Health Technol Assess. 2015;19(14).

30. Royston P, Parmar MK. Flexible parametric proportional-hazards and proportional-odds models for censored survival data, with application to prognostic modelling and estimation of treatment effects. Stat Med. 2002;21(15):2175-97.

31. Dewar R, Khan IJ. A new SAS macro for flexible parametric survival modeling: applications to clinical trials and surveillance data. Clin Invest. 2015;5(12):855-66.

32. Husereau D, Drummond M, Petrou S, et al. Consolidated health economic evaluation reporting standards (CHEERS) statement. Cost Effectiveness Resour Alloc. 2013;11(1):6.

33. Davies M, Quinnell T, Oscroft N, et al. Hospital outcomes and long-term survival after referral to a specialized weaning unit. $\mathrm{Br}$ J Anaesth. 2017;118(4):563-9.

34. Depuydt P, Oeyen S, De Smet S, et al. Long-term outcome and health-related quality of life in difficult-to-wean patients with and without ventilator dependency at ICU discharge: a retrospective cohort study. BMC Pulm Med. 2016;16(1):133.

35. Pilcher D, Bailey M, Treacher D, et al. Outcomes, cost and long term survival of patients referred to a regional weaning centre. Thorax. 2005;60(3):187-92.

36. Khan I. Design \& analysis of clinical trials for economic evaluation \& reimbursement: an applied approach using SAS \& STATA. 1st ed. New York: Chapman and Hall/CRC; 2015.

37. Keenan SP, Gregor J, Sibbald WJ, et al. Noninvasive positive pressure ventilation in the setting of severe, acute exacerbations of chronic obstructive pulmonary disease: more effective and less expensive. Crit Care Med. 2000;28(6):2094-102.

38. Dritsaki M, Achana F, Mason J, et al. Methodological issues surrounding the use of baseline health-related quality of life data to inform trial-based economic evaluations of interventions within emergency and critical care settings: a systematic literature review. PharmacoEconomics. 2017;35(5):501-15. 